\title{
Delay in reviewing test results prolongs hospital length of stay: a retrospective cohort study
}

\author{
Mei-Sing Ong ${ }^{1 *}$, Farah Magrabi $^{2}$ and Enrico Coiera ${ }^{2}$
}

\begin{abstract}
Background: Failure in the timely follow-up of test results has been widely documented, contributing to delayed medical care. Yet, the impact of delay in reviewing test results on hospital length of stay (LOS) has not been studied. We examine the relationship between laboratory tests review time and hospital LOS.

Methods: A retrospective cohort study of inpatients admitted to a metropolitan teaching hospital in Sydney, Australia, between 2011 and $2012(n=5804)$. Generalized linear models were developed to examine the relationship between hospital LOS and cumulative clinician read time (CRT), defined as the time taken by clinicians to review laboratory test results performed during an inpatient stay after they were reported in the computerized test reporting system. The models were adjusted for patients' age, sex, and disease severity (measured by the Charlson Comorbidity index), the number of test panels performed, the number of unreviewed tests pre-discharge, and the cumulative laboratory turnaround time (LTAT) of tests performed during an inpatient stay.

Results: Cumulative CRT is significantly associated with prolonged LOS, with each day of delay in reviewing test results increasing the likelihood of prolonged LOS by $13.2 \%(p<0.0001)$. Restricting the analysis to tests with abnormal results strengthened the relationship between cumulative CRT and prolonged LOS, with each day of delay in reviewing test results increasing the likelihood of delayed discharge by $33.6 \%(p<0.0001)$. Increasing age, disease severity and total number of tests were also significantly associated with prolonged LOS. Increasing number of unreviewed tests was negatively associated with prolonged LOS.

Conclusions: Reducing unnecessary hospital LOS has become a critical health policy goal as healthcare costs escalate. Preventing delay in reviewing test results represents an important opportunity to address potentially avoidable hospital stays and unnecessary resource utilization.
\end{abstract}

Keywords: Length of stay, Test results, Delay

\section{Background}

Inpatient hospital services constitute almost one-third of all health care expenditures in the United States [1]. Substantial research and policy efforts have been directed at reducing unnecessary hospital length of stay (LOS), a key indicator for inpatient resource use. Research into the determinants of hospital length of stay (LOS) has identified a range of patient characteristics and clinical predictors contributing to prolonged LOS

\footnotetext{
* Correspondence: Mei-Sing_Ong@hms.harvard.edu

${ }^{1}$ Department of Population Medicine, Harvard Medical School and Harvard Pilgrim Health Care Institute, 401 Park Drive, Suite 401E, Boston, MA 02115, USA

Full list of author information is available at the end of the article
}

[2]. These include age, sex, disease severity, presenting conditions and complications [3-10]. While useful in guiding resource planning, most patient and diseasespecific variables are not amenable to interventions that would reduce unnecessary hospital stays.

More often than not, hospital LOS is also driven by institutional factors unrelated to individual patients' conditions, such as clinical workflow and hospital resources. Patients may experience prolonged hospitalizations due to delays in accessing care [11]. Identifying these modifiable factors can provide important opportunities for interventions.

(c) The Author(s). 2018 Open Access This article is distributed under the terms of the Creative Commons Attribution 4.0 International License (http://creativecommons.org/licenses/by/4.0/), which permits unrestricted use, distribution, and reproduction in any medium, provided you give appropriate credit to the original author(s) and the source, provide a link to the Creative Commons license, and indicate if changes were made. The Creative Commons Public Domain Dedication waiver (http://creativecommons.org/publicdomain/zero/1.0/) applies to the data made available in this article, unless otherwise stated. 
Here, we conduct the first study to explore the relationship between laboratory tests review time and LOS. Because clinical laboratory testing is an integral part of healthcare delivery, driving a large proportion of critical medical decisions, we hypothesize that delay in reviewing test results prolongs hospital LOS.

\section{Methods}

\section{Settings and participants}

This study was conducted at a 370-bed metropolitan teaching hospital. A hospital-wide computerized system was in place for reporting all diagnostic tests. The system captured detailed information on the time and date when laboratory tests were requested, when the results were available for review, and when they were viewed by the providers. Participants included all inpatients admitted to the hospital between February 2011 and February 2012. Clinical pathology tests performed were extracted from the computerized test-reporting system; these included clinical chemistry, clinical pharmacology, hematology, immunology and microbiology. Tests relating to arterial blood gases were excluded, since the results for these tests were communicated directly to the ordering physician. We further extracted from the hospital admission database the admission details of the patients, including date of admission and discharge, principal diagnosis (expressed as free text), and the department where inpatient care was provided. This study was approved by the Human Research Ethics Committee at the University of New South Wales (Sydney, Australia), with patient consent waived.

\section{Study outcomes}

The primary outcome of interest was the relationship between hospital LOS and clinician read time (CRT), defined as the time taken by a provider to access laboratory test results after they were available for review in the test reporting system (Fig. 1). The provider who reviewed the test results may be the ordering provider or any members of the healthcare team. Since patients often underwent multiple tests, we calculated the cumulative CRT for all tests performed during an inpatient stay.
Timeliness in reviewing test results is dependent on timely processing of laboratory tests. The impact of laboratory turnaround time (LTAT), defined as the time between ordering of test and when the test results are available for review, has been reported to prolong LOS in high-volume patient care settings such as the emergency department. We therefore assessed the modifying effect of LTAT on the relationship between CRT and hospital LOS, and compare the effects of CRT and LTAT on hospital LOS. We hypothesize that cumulative CRT is independently associated with prolonged hospital LOS.

\section{Statistical analysis}

Descriptive statistics were used to quantify the prevalence of baseline characteristics, including patient demographics, inpatient medical specialties, the time of day tests were ordered, processed and reviewed. The impact of CRT on LOS was evaluated using generalized linear models. We used the gamma family of distributions to account for skewness in the distribution of LOS, with logarithm as the link function. We included in the model patients' age, sex, and disease severity (measured by the Charlson Comorbidity index), the total number of test panels performed during the inpatient stay, and the cumulative LTAT. We further controlled for the number of tests that were not reviewed during the inpatient stay, since tests that were not followed up were unlikely to prolong hospital LOS. An additional sensitivity analysis was performed that excluded patients with tests that were not followed up during an inpatient stay. To compare the impact of delay in reviewing abnormal test results and normal test results, we conducted a sensitivity analysis that considered only tests with abnormal findings, and a separate analysis that included only normal test results. With the exception of sex, all variables were modeled as continuous variables. The resulting estimated coefficients are presented as backtransformed (exponentiated), which are interpretable as the multiplicative effects of predictor variables on the expected LOS.

To address potential reverse causation in the association between CRT and LOS, whereby prolonged LOS causes delay in CRT, we assessed variability in the

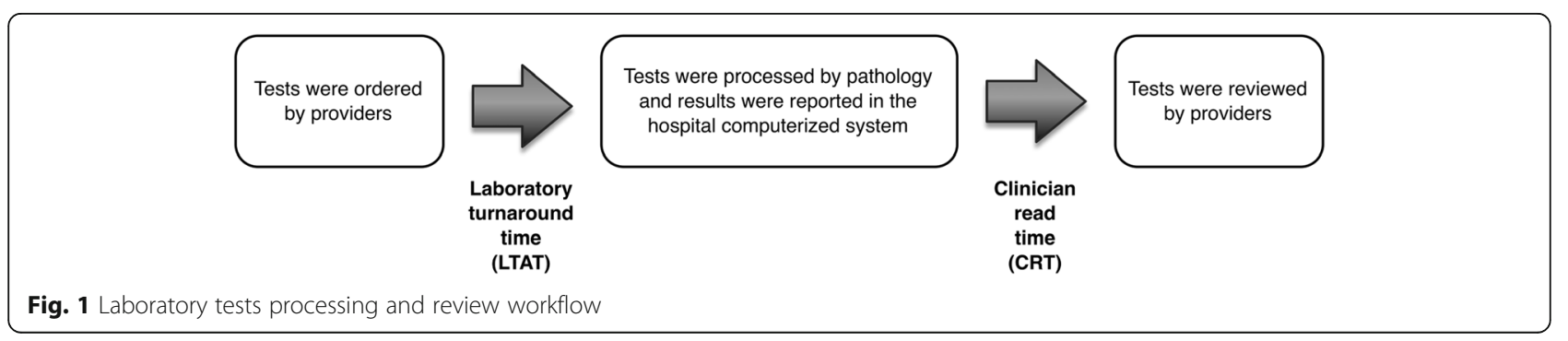


follow-up behavior of providers throughout inpatient stays, by quantifying the average CRT for tests ordered on each day of hospital admission, from day one to day 14 of admission. We observed that tests ordered on the first 2 days of admission had an average CRT of 26 and $21 \mathrm{~h}$, respectively (Additional file 1: Figure S1). The average CRT for tests ordered on subsequent days (i.e. day three to 14 of admission) was about $12 \mathrm{~h}$ and did not vary with prolonged LOS. To account for this pattern, we conducted an additional sensitivity analysis where CRT was measured based only on tests ordered in the first $48 \mathrm{~h}$ of admission, and a further analysis that quantified CRT based on tests ordered after the first $48 \mathrm{~h}$ of admission among patients who were hospitalized for two or more days.

Analyses were conducted using $\mathrm{R}$ statistical software version 3.4.3. All tests of statistical significance were two-tailed and used an $\alpha$ level of $p<0.05$.

\section{Subgroup analyses}

Clinical workflow and resources may differ across departments within the hospital, which may alter the relationship between CRT and hospital LOS. To assess if the effect of CRT on hospital LOS was consistently observed across different medical departments, we performed subgroup analyses for four of the departments with the largest number of inpatient admissions during the study period: geriatrics, cardiology, general surgery, and psychiatry. Separate models were developed for each department to assess the relationship between cumulative CRT and LOS. To account for potential confounding associated with patient heterogeneity, we further performed additional subgroup analyses that considered inpatients with the same principal admitting diagnosis. Two subgroups were selected a priori: patients with renal failure and patients who underwent elective knee or hip replacement surgery; the former represents a group of critically ill patients requiring urgent care, and the latter represents a more routine and non-urgent patient population. We hypothesize that the impact of CRT on LOS was greater among the renal failure population, compared with patients who were admitted for an elective knee or hip replacement surgery.

\section{Results}

A total of 5804 inpatient admissions satisfied the inclusion criteria (Table 1). The mean and median LOS were 7.5 and 4 days, respectively. The distribution of LOS is illustrated in Fig. 2a. On average, 7.2 test panels were performed during an inpatient stay, with a median of 4 test panels per inpatient stay. An inpatient experienced a mean cumulative CRT of 1.6 days, and a cumulative LTAT of 2.5 days. The average CRT and LTAT for
Table 1 Study participants $(n=5804)$

\begin{tabular}{|c|c|}
\hline Attributes & N (\%) \\
\hline \multicolumn{2}{|l|}{ Age } \\
\hline$<=30$ & $140(2.4)$ \\
\hline 31 to 64 & $2530(43.6)$ \\
\hline$>=65$ & $3134(54.0)$ \\
\hline Sex (female) & $2481(42.7)$ \\
\hline \multicolumn{2}{|l|}{ Charlson comorbidity index } \\
\hline 0 & $3508(60.4)$ \\
\hline 1 & $897(15.5)$ \\
\hline 2 & $649(11.2)$ \\
\hline 3 & $318(5.5)$ \\
\hline$>=4$ & $432(7.4)$ \\
\hline Patients with one or more unreviewed tests, n (\%) & $3129(53.9)$ \\
\hline \multicolumn{2}{|l|}{ Department, n (\%) } \\
\hline Geriatrics & $983(16.9)$ \\
\hline Cardiology & $765(13.2)$ \\
\hline General surgery & $554(9.5)$ \\
\hline Psychiatry & $396(6.8)$ \\
\hline Respiratory medicine & $388(6.7)$ \\
\hline Orthopedic surgery & $354(6.1)$ \\
\hline Neurology & $237(4.1)$ \\
\hline Gastroenterology & $217(3.7)$ \\
\hline Vascular surgery & $155(2.7)$ \\
\hline Neurosurgery & $150(2.6)$ \\
\hline Hematology & $147(2.5)$ \\
\hline Urology & $144(2.5)$ \\
\hline Cardiothoracic surgery & $108(1.9)$ \\
\hline Nephrology & $102(1.8)$ \\
\hline Dermatology & $75(1.3)$ \\
\hline Rehabilitation & $68(1.2)$ \\
\hline Otolaryngology & $62(1.1)$ \\
\hline Palliative care & $57(1.0)$ \\
\hline Drug and alcohol & $53(0.9)$ \\
\hline Immunology & $50(0.9)$ \\
\hline Infectious diseases & $49(0.8)$ \\
\hline Endocrinology & $32(0.6)$ \\
\hline Oncology & $29(0.5)$ \\
\hline Obstetrics and gynecology & $23(0.4)$ \\
\hline Rheumatology & $23(0.4)$ \\
\hline Maxillofacial surgery & $6(0.1)$ \\
\hline Dental surgery & $2(0.03)$ \\
\hline Radiation oncology & $2(0.03)$ \\
\hline Ophthalmology & $1(0.02)$ \\
\hline Unspecified & $572(9.9)$ \\
\hline
\end{tabular}



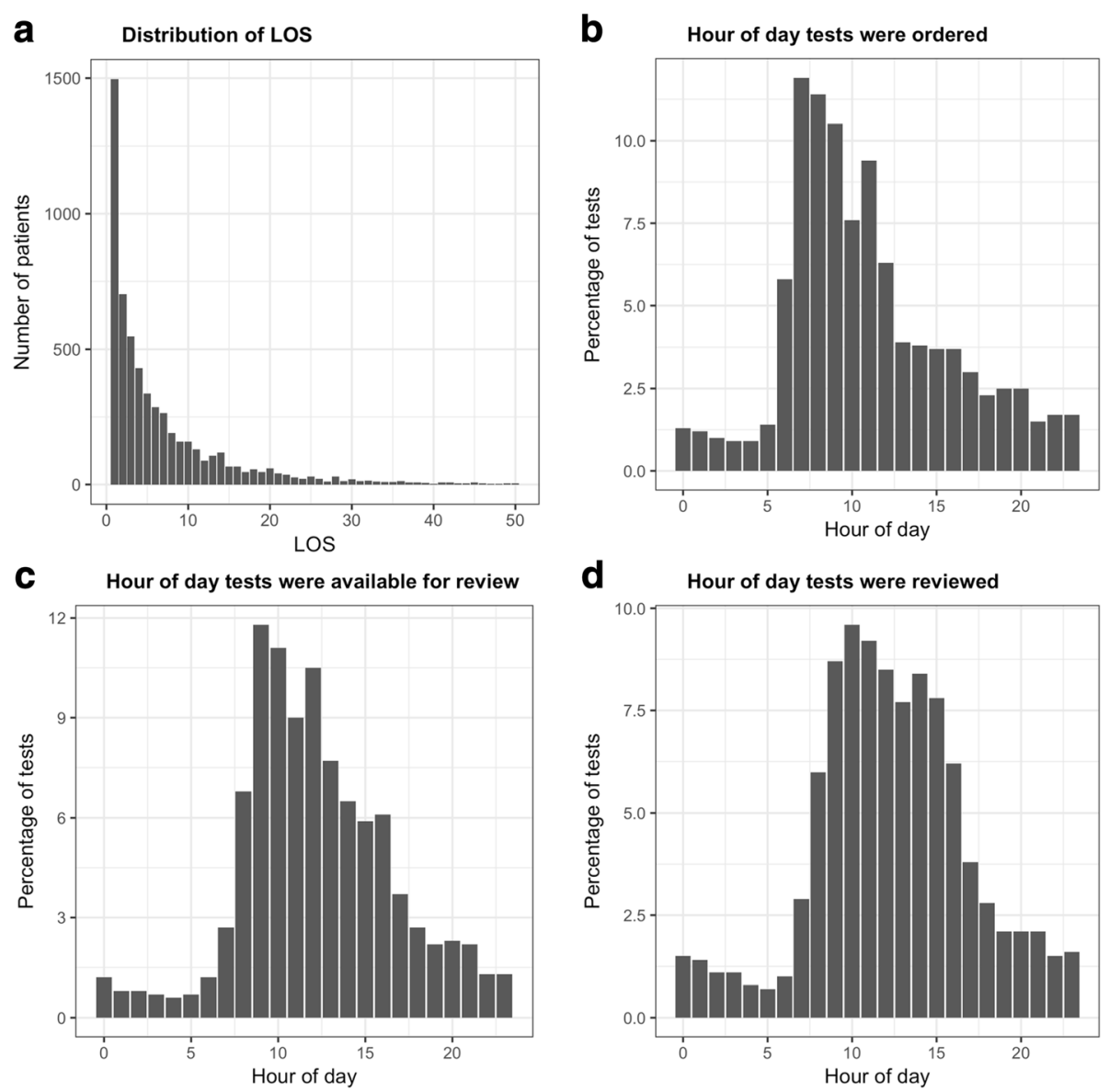

Fig. 2 Distribution of hospital length of stay (LOS) and patterns of test ordering, processing and review

individual tests was $12.7 \mathrm{~h}$ and $16.1 \mathrm{~h}$, respectively; the median CRT and LTAT for individual tests was an hour.

Cumulative CRT is significantly associated with prolonged LOS, with each day of delay in reviewing test results increasing the likelihood of prolonged hospital stay by $13.2 \%(p<0.0001)$. Increasing age, disease severity and total number of tests were also significantly associated with prolonged LOS (Table 2a). Patient's sex was not associated with LOS in univariate analysis, and was therefore excluded from the multivariate models. While cumulative LTAT was significantly associated with LOS in univariate analysis, its effect on LOS became insignificant in the multivariate generalized linear model. Increasing number of unreviewed tests was negatively associated with prolonged LOS.

A total of 3129 (53.9\%) patients had one or more test results that were not followed up during hospitalization. Excluding these patients from the analysis strengthened the impact of cumulative CRT on prolonged LOS, with each day of delay in reviewing test results increasing the likelihood of delayed discharge by $18.6 \%(p<0.0001)$. In this subpopulation, increasing age and disease severity were not associated with prolonged LOS; increasing number of test panels and cumulative LTAT were associated with prolonged LOS (Table $2 \mathrm{~b}$ ).

Of the laboratory tests ordered, $30.4 \%$ had abnormal results. Including only tests with abnormal results in the analysis strengthened the relationship between cumulative CRT and prolonged LOS, with each day of delay in reviewing test results increasing the likelihood of delayed discharge by $33.6 \%(p<0.0001)$, compared with $12.2 \%(p<0$. 0001) when only tests with normal results were considered (Table 2c, d). In the two sensitivity analyses that quantified delay based on tests ordered within the first $48 \mathrm{~h}$ of admission and tests ordered after the first $48 \mathrm{~h}$ of admission, respectively, cumulative CRT remained a significant predictor of LOS $(p<0.0001)$ (Table 2e, f).

\section{Subgroup analyses}

The effect of cumulative CRT on prolonged LOS was consistently observed across different departments (geriatrics, cardiology, general surgery and psychiatry) and patient subgroups with varying principal diagnoses (renal failure, knee or hip replacement) (Table 3). 
Table 2 Generalized linear model for predicting hospital LOS

\begin{tabular}{lcl}
\hline Covariate & Estimate $(\beta)(95 \%$ Cl) & $p$-value \\
\hline (a) Primary analysis including all inpatients & \\
Age & $1.003(1.001-1.005)$ & $<0.0001$ \\
Charlson comorbidity index & $1.020(1.004-1.037)$ & 0.017 \\
Number of test panels & $1.054(1.048-1.060)$ & $<0.0001$ \\
Cumulative CRT (days) & $1.132(1.116-1.149)$ & $<0.0001$ \\
Cumulative LTAT (days) & $0.996(0.988-1.004)$ & 0.165 \\
Number of unreviewed tests & $0.988(0.985-0.992)$ & $<0.0001$ \\
(b) Sensitivity analysis including only patients without any unreviewed \\
tests & $1.001(0.999-1.004)$ & 0.267 \\
Age & $1.019(0.989-1.051)$ & 0.247 \\
Charlson comorbidity index & $1.060(1.047-1.073)$ & $<0.0001$ \\
Number of test panels & $1.186(1.154-1.221)$ & $<0.0001$ \\
Cumulative CRT (days) & $1.028(1.004-1.054)$ & 0.022 \\
Cumulative LTAT (days) &
\end{tabular}

(c) Sensitivity analysis including only laboratory tests with abnormal results

$\begin{array}{lll}\text { Age } & 1.001(0.999-1.002) & 0.381 \\ \text { Charlson comorbidity index } & 1.020(1.004-1.037) & 0.022 \\ \text { Number of test panels } & 1.073(1.065-1.081) & <0.0001 \\ \text { Cumulative CRT (days) } & 1.336(1.294-1.380) & <0.0001 \\ \text { Cumulative LTAT (days) } & 1.010(0.993-1.029) & 0.231 \\ \text { Number of unreviewed tests } & 0.971(0.958-0.984) & <0.0001\end{array}$

(d) Sensitivity analysis including only laboratory tests with normal results

$\begin{array}{lll}\text { Age } & 1.003(1.001-1.004) & 0.002 \\ \text { Charlson comorbidity index } & 1.019(1.003-1.036) & 0.026 \\ \text { Number of test panels } & 1.063(1.056-1.070) & <0.0001 \\ \text { Cumulative CRT (days) } & 1.122(1.105-1.139) & <0.0001 \\ \text { Cumulative LTAT (days) } & 0.990(0.982-0.999) & 0.002 \\ \text { Number of unreviewed tests } & 0.984(0.980-0.988) & <0.0001\end{array}$

(e) Sensitivity analysis including only tests ordered in the first two days of admission

$\begin{array}{lll}\text { Age } & 1.003(1.001-1.005) & 0.002 \\ \text { Charlson comorbidity index } & 1.018(1.001-1.037) & 0.057 \\ \text { Number of test panels } & 1.065(1.058-1.072) & <0.0001 \\ \text { Cumulative CRT (days) } & 1.030(1.017-1.044) & <0.0001 \\ \text { Cumulative LTAT (days) } & 1.017(1.008-1.027) & <0.0001 \\ \text { Number of unreviewed tests } & 0.987(0.983-0.991) & <0.0001\end{array}$

(f) Sensitivity analysis excluding tests ordered in the first two days of admission

\begin{tabular}{lll} 
Age & $1.001(1.000-1.003)$ & 0.159 \\
Charlson comorbidity index & $0.999(0.984-1.013)$ & 0.855 \\
Number of test panels & $1.025(1.020-1.031)$ & $<0.0001$ \\
Cumulative CRT (days) & $1.055(1.046-1.064)$ & $<0.0001$ \\
Cumulative LTAT (days) & $0.990(0.983-0.997)$ & 0.001 \\
Number of unreviewed tests & $0.989(0.986-0.992)$ & $<0.0001$ \\
\hline
\end{tabular}

Table 3 Subgroup analyses by department and principal diagnoses

\begin{tabular}{lll}
\hline Covariate & Estimate $(\beta)(95 \% \mathrm{Cl})$ & $p$-value \\
\hline (a) Department: geriatric medicine & & \\
Age & $0.998(0.991-1.005)$ & 0.516 \\
Charlson comorbidity index & $0.992(0.957-1.029)$ & 0.661 \\
Number of test panels & $1.072(1.060-1.085)$ & $<0.0001$ \\
Cumulative CRT (days) & $1.108(1.080-1.137)$ & $<0.0001$ \\
Cumulative LTAT (days) & $0.995(0.972-1.018)$ & 0.652 \\
Number of unreviewed tests & $0.996(0.990-1.003)$ & 0.274
\end{tabular}

(b) Department: cardiology medicine

$1.001(0.998-1.004) \quad 0.467$

Charlson comorbidity index

$1.049(1.018-1.083)$

0.002

Number of test panels

$1.112(1.099-1.126)$

$<0.0001$

Cumulative CRT (days)

1.049 (1.012-1.092)

0.001

Cumulative LTAT (days)

0.979 (0.962-0.998)

0.003

Number of unreviewed tests

$0.999(0.993-1.006)$

0.829

(c) Department: general surgery

Age

1.003 (0.999-1.006)

0.123

Charlson comorbidity index

1.019 (0.986-1.056)

0.283

Number of test panels

1.114 (1.097-1.132)

$<0.0001$

Cumulative CRT (days)

1.034 (1.000-1.076)

0.026

Cumulative LTAT (days)

$0.975(0.944-1.008)$

0.133

Number of unreviewed tests

$0.992(0.986-0.998)$

0.012

(d) Department: psychiatry

Age

$1.011(1.000-1.023)$

0.054

Charlson comorbidity index

$1.060(0.951-1.212)$

0.368

Number of test panels

$1.168(1.080-1.270)$

$<0.0001$

Cumulative CRT (days)

$1.140(1.077-1.213)$

$<0.0001$

Cumulative LTAT (days)

$1.048(0.995-1.112)$

0.031

Number of unreviewed tests

$0.959(0.942-0.981)$

0.0002

(e) Principal diagnosis: renal failure

Age

1.007 (0.995-1.019)

0.289

Charlson comorbidity index

$0.921(0.841-1.013)$

0.087

Number of test panels

$1.037(0.995-1.080)$

0.090

Cumulative CRT (days)

$1.182(1.092-1.289)$

0.0002

Cumulative LTAT (days)

1.006 (0.966-1.050)

0.776

Number of unreviewed tests

0.994 (0.977-1.013)

0.509

(f) Principal diagnosis: knee or hip replacement surgery

Age

1.004 (0.994-1.014)

0.404

Charlson comorbidity index

$1.025(0.848-1.265)$

0.815

Number of test panels

$1.054(1.016-1.095)$

0.007

Cumulative CRT (days)

$1.177(1.071-1.299)$

0.003

Cumulative LTAT (days)

1.004 (0.905-1.116)

0.941

Number of unreviewed tests

$0.992(0.978-1.008)$

0.335 
Increasing number of tests performed during inpatient stay was also consistently associated with prolonged LOS. The relationship between prolonged LOS and the remaining covariates were variably observed across departments and principal diagnoses.

\section{Clinical workflow}

The majority of inpatient laboratory tests were ordered between 6 am and $12 \mathrm{pm}$ (Fig. 2b), and most test results were available for review between 8 am and 2 pm (Fig. 2c). Review of test results followed the same trend, peaking between 9 am and 3 pm (Fig. 2d).

Figure 3 depicts the relationship between the time of day a laboratory test was placed, and the average CRT and LTAT, considering only tests that were reviewed during the inpatient stay. Both CRT and LTAT appeared to peak at around $2 \mathrm{pm}$, where the average total time taken to process and review a test was $23.7 \mathrm{~h}$ after a test was ordered. Comparing the CRT and LTAT of laboratory tests ordered before and after $2 \mathrm{pm}$, those that were placed after $2 \mathrm{pm}$ experienced a much greater total delay $(18.0 \mathrm{~h}$ vs $12.4 \mathrm{~h} ; p<0.0001)$, with both a greater CRT (7.5 vs $5.2 \mathrm{~h} ; p<0.0001)$ and LTAT (10.1 vs $6.8 \mathrm{~h} ; p<0.0001)$.

\section{Discussion}

We established for the first time that delay in reviewing laboratory test results is significantly associated with prolonged hospital LOS. Delay in reviewing test results may prevent timely diagnosis and treatment, resulting in suboptimal outcomes that may further contribute to prolonged LOS. In our study, only one-third of test results had abnormal findings. Thus, in most cases, delay in test results follow-up was unlikely to affect health outcomes. However, our analysis shows that delay in reviewing normal test results is also a significant

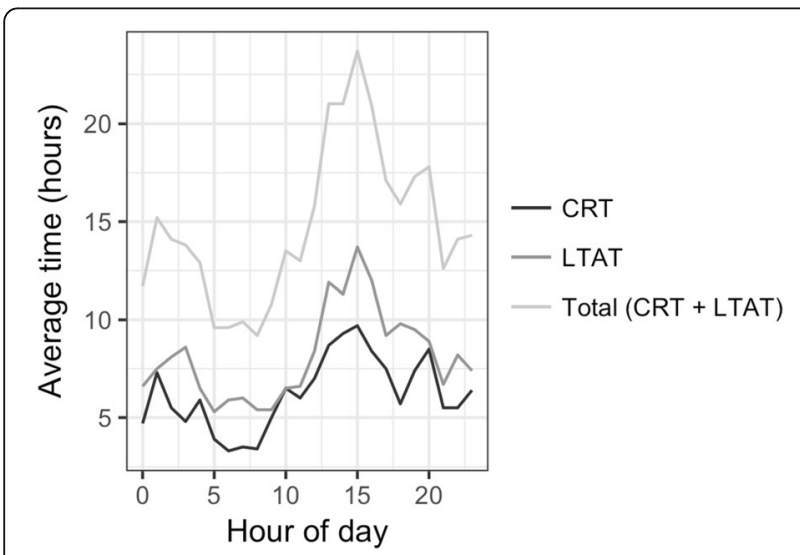

Fig. 3 The relationship between hour of day tests were ordered, and the laboratory turnaround time (LTAT), clinician read time (CRT), and the total time (LTAT + CRT) predictor of prolonged hospitalization. Patients may remain hospitalized while awaiting their test results to be reviewed before a discharge decision can be made. Reducing test review delay therefore represents an important opportunity to address potentially avoidable hospital stays and unnecessary resource utilization.

Turnaround time for laboratory tests did not appear to affect hospital LOS in the primary analysis that included all inpatients. A probable explanation is that a large proportion of laboratory tests were not actually reviewed prior to patient discharge [12] - delay in processing these tests were therefore unlikely to influence LOS. Indeed, the number of unreviewed tests was found to be negatively associated with LOS. In the secondary analysis that considered only patients who had their test results reviewed during their inpatient stay, a significant relationship between increasing cumulative LTAT and prolonged LOS was observed. However, the strength of the association was notably weaker than the association between delay in reviewing test results and LOS. The effects of LTAT on LOS have been extensively studied, primarily in the emergency care setting [13]. While a number of studies demonstrated a significant relationship between LTAT and LOS, others have failed to show the benefit of reducing LTAT on LOS $[14,15]$. Substantial efforts to improve the efficiency of pathology workflow has significantly reduced the delay associated with laboratory turnaround time. However, delay in reviewing test results has received much less attention. Our analysis suggests that unless test results are reviewed in a timely manner, any benefits gained from reducing LTAT will be greatly diminished.

The time of day laboratory tests were ordered, processed and reviewed followed similar trends, increasing in volume during late mornings and early afternoons, and tapering off during late-afternoon (Fig. 2b-d). This likely reflects the window of time whereby the bulk of routine clinical decision-making took place. We further observed that the average CRT and LTAT for individual tests varied by the time of day the tests were ordered, peaking at around $2 \mathrm{pm}$, coinciding with the time with the highest volume of tests ordered (Fig. 3). The high load of laboratory tests may have caused the delay in turnaround time, and as a result, laboratory tests that were ordered after $2 \mathrm{pm}$ had a substantially longer delay in CRT and LTAT. These findings strongly suggest that clinical workflow and available resources played an important role in influencing when test results were processed and reviewed. Improving workflow and resource allocation may therefore offer an opportunity to reduce unnecessary hospital stay. For example, enhancing capacity planning to address variability in staffing workload, such as increasing resources during peak periods [16], and reviewing test results when they are 
available and discharging patients when they are clinically ready for discharge, regardless of the time of day, may improve patient flow and reduce unnecessary LOS. There are also potential opportunities for leveraging health informatics technology to facilitate the timely notification of available test results to providers more quickly. Any such interventions would need to ensure that providers are not unduly burdened with alarm fatigue and additional workload, and that the interventions do not lead to other unintended consequences.

\section{Study limitations}

Our study is limited to a single center. Other centers may vary greatly in resources and organizational structure, which may in turn influence the relationship between delay in reviewing test results and LOS. Nonetheless, we believe that the organization of care in this teaching hospital is similar to other hospitals in most developed countries. To address the potential bias caused by variability in clinical workflow, we performed subgroup analyses of departments that served vastly different patient subpopulations within the hospital, including geriatrics, general surgery, cardiology and psychiatry. Additionally, subgroup analyses that considered patients with different principal diagnosis were conducted. The relationship between delay in test results follow-up and LOS was consistently observed in these analyses. Indeed, cumulative CRT and the number of tests performed were the only variables that were significantly associated with LOS across all settings and patient populations.

Our study cannot establish causality. While we have included potential confounders in our models, including disease severity and patient's age, we could not account for other important factors such as staffing level, team rotations, and the behavior of individual providers these factors can lead to delay in test results follow-up and directly impact the quality of care that results in prolonged hospitalization independent of delay in test results follow-up. Providers may also be less inclined to rapidly review test results if they expect patients to remain hospitalized due to their conditions. However, our analysis of the follow-up behavior of test results by day of admission suggests that on average, the overall test results follow-up patterns were not driven by the expected LOS. Furthermore, in sensitivity analyses that considered separately tests ordered within the first 2 days of admission and tests ordered after the first 2 days of admission, the association between delay in test results follow-up and LOS remained significant.

There is also limitation in the use of Charlson comorbidity index to adjust for disease severity, an important determinant of LOS. While Charlson comorbidity index - a weighted score of comorbidities based on their relative risk of mortality - has been extensively validated in published literature as a reliable prognostic indicator for mortality [17-20], its predictive power may vary across different patient subpopulations. To address this limitation, we conducted additional subgroup analyses for patients who were likely to have similar disease severity based on their principal reason for admission, including patients with renal failure and those who were admitted for an elective hip or knee replacement. In all analyses, the relationship between test follow-up delay and prolonged LOS was consistently observed. While further research is needed to understand the relationship between delay in reviewing test results and LOS and to account for unmeasured confounders, our unique data source that captures fine-grained timestamps of when tests were ordered, processed and reviewed, offer the rare opportunity to quantify delay in test results follow-up and to study its impact on LOS.

\section{Conclusions}

Reducing unnecessary hospital LOS has become a critical health policy goal as the costs of healthcare escalate, and budgetary constraints in health care limit available resources. Our study highlights an important, yet underexplored, risk factor of prolonged LOS that warrants further investigation. Preventing delay in reviewing test results may provide a low-hanging fruit for ameliorating unnecessary hospital stays.

\section{Additional file}

Additional file 1: Figure S1. Average clinical read time (CRT) for tests ordered on a given day of admission. (DOCX $100 \mathrm{~kb}$ )

\section{Abbreviations \\ CRT: Clinician read time; LOS: Length of stay; LTAT: Laboratory turnaround time}

\section{Acknowledgments}

We thank the study site for providing the data source used in this study, and Dr. Fang Zhang (Harvard Medical School and Harvard Pilgrim Healthcare) for his guidance on power analysis.

\section{Availability of data and materials \\ The data that support the findings of this study are available from the University of New South Wales, but restrictions apply to the availability of these data, which were used under license for the current study, and so are not publicly available. Data are however available from the authors upon reasonable request and with permission of University of New South Wales and the affiliated hospital.}

\section{Authors' contributions}

All authors made substantial contribution to the conception and design of the study, and interpretation of data. MO performed the study analysis and drafted the manuscript; EO and FM revised the manuscript and gave final approval of the version to be published. All authors had full access to the data in the study and take responsibility for the integrity of the data and the accuracy of the data analysis. 


\section{Ethics approval and consent to participate}

This study was approved by the Human Research Ethics Committee at the University of New South Wales (Sydney, Australia), with patient consent waived.

\section{Competing interests}

The authors declare that they have no competing interests.

\section{Publisher's Note}

Springer Nature remains neutral with regard to jurisdictional claims in published maps and institutional affiliations.

\section{Author details}

'Department of Population Medicine, Harvard Medical School and Harvard Pilgrim Health Care Institute, 401 Park Drive, Suite 401E, Boston, MA 02115, USA. ${ }^{2}$ Australian Institute of Health Innovation, Macquarie University, Sydney, Australia.

Received: 24 October 2017 Accepted: 3 May 2018

Published online: 16 May 2018

\section{References}

1. Gonzalez JM. National Health Care Expenses in the U.S. civilian noninstitutionalized population, 2011. MEPS statistical brief no. 425. Rockville, MD: Agency for Healthcare Research and Quality; 2013.

2. Lu M, Sajobi T, Lucyk K, Lorenzetti D, Quan H. Systematic review of risk adjustment models of hospital length of stay (LOS). Med Care. 2015 Apr; 53(4):355-65.

3. Carey M, Sheth $\mathrm{H}$, Braithwaite R. A prospective study of reasons for prolonged hospitalizations on a general medicine teaching service. J Gen Intern Med. 2005;20:108-15.

4. Geissler A, Scheller-Kreinsen D, Quentin W. Do diagnosis-related groups appropriately explain variations in costs and length of stay of hip replacement? A comparative assessment of DRG systems across 10 European countries. Health Econ. 2012;21:103-15.

5. Ghali WA, Hall RE, Ash AS, et al. Identifying pre- and postoperative predictors of cost and length of stay for coronary artery bypass surgery. Am J Med Qual. 1999;14:248-54.

6. Every NR, Spertus J, Fihn SD, et al. Length of hospital stay after acute myocardial infarction in the myocardial infarction triage and intervention (MITI) project registry. J Am Coll Cardiol. 1996;28:287-93.

7. lezzoni LI, Shwartz M, Ash AS, et al. Does severity explain difference in hospital length of stay for pneumonia patients? Health Serv Res. 1996;1:65-76.

8. Matsui K, Goldman L, Johnson PA, et al. Comorbidity as a correlate of length of stay for hospitalized patients with acute chest pain. J Gen Intern Med. 1996;11:262-8.

9. Rochon PA, Katz JA, Morrow LA, et al. Comorbid illness is associated with survival and length of hospital stay in patients with chronic disability: a prospective comparison of three comorbidity indices. Med Care. 1996;34: 1093-101.

10. Kieszak S, Flanders D, Kosinski A, et al. A comparison of the Charlson comorbidity index derived from medical record data and administrative billing data. J Clin Epidemiol. 1999;52:137-42.

11. Weissman JS, Stern R, Fielding SL, Epstein AM. Delayed access to healthcare: risk factors, reasons, and consequences. Ann Intern Med. 1991;114(4):325-31.

12. Ong MS, Magrabi F, Jones G, Coiera E. Last orders: follow-up of tests ordered on the day of hospital discharge. Arch Intern Med. 2012; 172(17):1347-9.

13. Hawkins RC. Laboratory turnaround time. Clin Biochem Rev. 2007:28(4):179-94.

14. Bruins M, Oord H, Bloembergen P, Wolfhagen M, Casparie A, Degener J, Ruijs $G$. Lack of effect of shorter turnaround time of microbiological procedures on clinical outcomes: a randomised controlled trial among hospitalised patients in the Netherlands. Eur J Clin Microbiol Infect Dis. 2005;24(5):305-13.

15. Saunders CE, Makens PK, Leblanc LJ. Modeling emergency department operations using advanced computer simulation systems. Ann Emerg Med. 1989;18:134-40.

16. Litvak E, Buerhaus PI, Davidoff F, Long MC, McManus ML, Berwick DM. Managing unnecessary variability in patient demand to reduce nursing stress and improve patient safety. Jt Comm J Qual Patient Saf. 2005; 31(6):330-8.
17. Quan H, Li B, Couris CM, Fushimi K, Graham P, Hider P, Januel JM Sundararajan $\mathrm{V}$. Updating and validating the Charlson comorbidity index and score for risk adjustment in hospital discharge abstracts using data from 6 countries. Am J Epidemiol. 2011;173(6):676-82.

18. Frenkel WJ, Jongerius EJ, Mandjes-van Uitert MJ, van Munster BC, de Rooij SE. Validation of the Charlson comorbidity index in acutely hospitalized elderly adults: a prospective cohort study. J Am Geriatr Soc. 2014;62(2):342-6.

19. Birim O, Kappetein AP, Bogers AJ. Charlson comorbidity index as a predictor of long-term outcome after surgery for nonsmall cell lung cancer. Eur J Cardiothorac Surg. 2005;28(5):759-62.

20. Tessier A, Finch L, Daskalopoulou SS, Mayo NE. Validation of the Charlson comorbidity index for predicting functional outcome of stroke. Arch Phys Med Rehabil. 2008:89(7):1276-83.

\section{Ready to submit your research? Choose BMC and benefit from:}

- fast, convenient online submission

- thorough peer review by experienced researchers in your field

- rapid publication on acceptance

- support for research data, including large and complex data types

- gold Open Access which fosters wider collaboration and increased citations

- maximum visibility for your research: over $100 \mathrm{M}$ website views per year

At BMC, research is always in progress.

Learn more biomedcentral.com/submissions 